\title{
Enquête Ethnobotanique Sur Les Plantes Médicinales Utilisées Dans La Prise En Charge Traditionnelle Des Maladies Infectieuses Dans La Région Sanitaire Lomé- Commune Du Togo
}

\author{
Aboudou, A. Dahounom (Master en Sciences Biomédicales) \\ Centre de Recherche et de Formation sur les Plantes Médicinales \\ (CERFOPLAM), Laboratoire de Physiologie et Pharmacologie, Faculté des \\ Sciences, Université de Lomé \\ Koffi Koudouvo (Maître de Conférences) \\ Centre de Recherche et de Formation sur les Plantes Médicinales \\ (CERFOPLAM), Laboratoire de Physiologie et Pharmacologie, Faculté des \\ Sciences, Université de Lomé
}

Doi:10.19044/esj.2021.v17n21p46

Submitted:17 July 2020

Accepted: 09 September 2020

Published: 30 June 2021
Copyright 2021 Author(s)

Under Creative Commons BY-NC-ND 4.0 OPEN ACCESS

Cite As:

Aboudou A.D. \& Koffi K. (2021). Enquête Ethnobotanique Sur Les Plantes Médicinales Utilisées Dans La Prise En Charge Traditionnelle Des Maladies Infectieuses Dans La Région Sanitaire Lomé-Commune Du Togo. European Scientific Journal, ESJ, 17(21), 46.

https://doi.org/10.19044/esj.2021.v17n21p46

\section{Résumé}

Afin de promouvoir la Médecine traditionnelle togolaise (MTT), préserver/conserver des savoirs et savoir-faire endogènes, une enquête ethnobotanique a été menée de Mai 2018 à Juillet 2019 pour recenser les recettes de plantes aux propriétés anti-infectieuses vendues dans les marchés de la Région Sanitaire Lomé-Commune du Togo.

ATRM(Achat en Triplet de Recettes Médicinales) a été la méthode de collecte des données. Les données recueillies qui portent sur les caractéristiques ethnopharmacologiques de ces recettes, ont été traitées et analysées avec des logiciels ethnobotaniques et statistiques appropriés afin de sélectionner quelques plantes pour de futurs tests de laboratoire.

Cent deux (102) recettes d'association de plantes constituées par 68 espèces appartenant à 37 familles, ont été recensées. Annonaceae et Leguminosae-Caesalpinioideae (5 espèces chacune), Apocynaceae, 
Euphorbiaceae, et Leguminosae-Papilionoideae (4 espèces chacune), ont été les familles les plus représentées. Anacardiaceae, Euphorbiaceae, et Leguminosae-Mimosoideae, avec respectivement 129, 75 et 63 citations, ont été les familles dont les espèces sont plus citées. Arbres (39,71\%), herbes (38,24\%), arbustes $(14,7 \%)$, lianes $(2,94 \%)$, arbrisseaux (2,94\%) et palmiers $(1,47 \%)$ ont été les types biologiques d'appartenance de ces espèces. Suivant les valeurs usuelles (VU), Lannea kerstingii ( $\mathrm{VU}=2,29)$, Bridelia ferruginea(VU=2,00) et Zanthoxylum xanthoxyloides( $V U=1,82)$ ont été les espèces plus usuelles. Ecorce de tronc $(53,57 \%)$, racine (16,13\%), tige feuillée (6,9\%), plante entière $(6,65 \%)$, fruit $(6,28 \%)$, et écorce de racine $(6,04 \%)$ ont été les parties les plus utilisées des espèces recensées qui sont préparées par décoction $(93.14 \%)$ et macération $(6,86 \%)$ puis administrées exclusivement par voie orale. Bridelia ferrugiea et Chamaechrista rotundifolia ont été sélectionnées pour de futurs tests antimicrobiens.

Ces résultats illustrent la richesse de la MTT en connaissances endogènes sur les plantes aux propriétés anti-infectieuses.

Mots clés: Phytomédecine, Plantes Médicinales Anti-Infectieuses, Herboristes De Marché, ATRM, Togo

\section{Ethnobotanical Survey On Medicinal Plants Used In The Traditional Management Of Infectious Diseases In The Lomé- Commune Health Region of Togo}

Aboudou, A. Dahounom (Master en Sciences Biomédicales)

Centre de Recherche et de Formation sur les Plantes Médicinales

(CERFOPLAM), Laboratoire de Physiologie et Pharmacologie, Faculté des

Sciences, Université de Lomé

Koffi Koudouvo (Maître de Conférences)

Centre de Recherche et de Formation sur les Plantes Médicinales

(CERFOPLAM), Laboratoire de Physiologie et Pharmacologie, Faculté des

Sciences, Université de Lomé

Abstract

In order to promote traditional Togolese medicine, to preserve and conserve endogenous knowledge and expertise, an ethnobotanical survey was carried out from May 2018 to July 2019 to record the recipes of plants with anti-infectious properties sold in the markets of the Lomé-Commune Health Region of Togo.

ATRM (Achat en Triplet de Recettes Médicinales) was the data collection method. The data collected about the ethnopharmacological 
characteristics of these recipes were treated and analyzed with appropriate ethnobotanical and statistical software for selection some plants for future laboratory tests.

One hundred and two associations of plant's recipes composited by 68 species belonging to 37 families were identified. Annonaceae and Leguminosae-Caesalpinioideae(5 species each), Apocynaceae, Euphorbiaceae, and Leguminosae-Papilionoideae(4 specie each) were the most represented families while Anacardiaceae(129 citations), Euphorbiaceae(75 citations) and Leguminosae-Mimosoideae(63 citations) were family of the most cited species. Trees(39.71\%), grasses(38.24\%), shrubs(14.7\%), lianas(2.94\%), bushes(2.94\%) and palms(1.47\%) were the different biological types to which these species belonged. According to the Used value(UV) Lannea kerstingii(UV=2.29), Bridelia ferruginea(UV=2.00) and Zanthoxylum xanthoxyloides(UV=1.82) were the most usual species. Stem bark(53.57\%), root(16.13\%), leafy stem(6.9\%), whole plant(6.65\%), fruit(6.28\%), and root bark(6.04\%) were the most commonly used parts of the listed species. These recipes which are prepared by decoction $(93.14 \%)$ and maceration(6.86\%) are administered exclusively by oral route. Bridelia ferrugiea and Chamaechrista rotundifolia were selected for laboratory tests to evaluate their antimicrobial activities in future.

Conclusion: These results illustrate the richness of traditional Togolese medicine in endogenous knowledge about anti-infectious' medicinal plants.

Keywords: Phytomedicine, Anti-Infectious Medicinal Plants, Market Herbalists, ATRM, Togo.

\section{Introduction}

Les recherches sur l'utilisation des plantes en Pharmacopée et Médecine Traditionnelle Africaines ont permis la découverte de nombreux principes actifs (molécules) de plusieurs médicaments utilisés aujourd'hui en Médecine moderne (Assouma, et al., 2018; Leonti et al., 2017; Soh et al., 2007; Cragg, et al., 2002). Parmi les molécules de ces médicaments, certaines ont été extraites directement des plantes médicinales, d'autres en sont des homologues structuraux. Nombreuses molécules traitant les maladies infectieuses y sont retrouvées (Dieye et al., 2020). Cependant, ces médicaments sont souvent très coûteux et peu supportables par les bourses des couches de population les plus pauvres surtout celles de l'Afrique subsaharienne, incapables de s'offrir de tels traitements. Cette situation, ajoutée aux résistances développées ces dernières années par les germes des maladies infectieuses et bactériennes contre les médicaments conventionnels selon Courvalin, 1997 et Dieye et al., 2020, ont contraint les populations de cette zone du monde à solliciter les plantes 
médicinales comme des alternatives de premier recours en soins de santé primaire.

Au Togo, dans la Région Sanitaire de Lomé-Commune(RSLC), des études ont signalé que plusieurs plantes sont utilisées traditionnellement par les populations pour traiter une diversité de maladies (Esseh et al., 2019; Denou et al., 2016; Koudouvo et al., 2011; Diallo et al., 2008; Adjanohoun et al., 1986). Cependant, peu ou rares sont les études qui ont portée sur les plantes aux propriétés anti-infectieuses vendues par les"' herboristes de marché’' du Togo (Koudouvo, 2009). La présente étude a été réalisée pour recenser par enquêtes ethnobotaniques, les plantes utilisées en médecine traditionnelle togolaise (MTT) dans la Région Sanitaire Lomé-Commune (RSLC) du Togo pour soigner les maladies infectieuses. La finalité de l'étude est d'établir la liste de cette catégorie de plantes médicinales de la zone en vue d'en sélectionner pour des tests sur des modèles de laboratoire afin de vérifier leurs activités pharmacologiques anti-infectieuses.

\section{Matériel et méthodes}

Zone d'étude

La RSLC qui est la zone d'étude, est située au sud du Togo à une latitude de $6^{\circ} 8.2488^{\prime}$ Nord et une longitude de $1^{\circ} 12.7362^{\prime}$ Est. Elle est limitée au Nord par la Région Maritime, au Sud par l'Océan Atlantique, à l'Est par le Bénin, à l'Ouest par le Ghana (Figure 1), et couvre une superficie de 8470 $\mathrm{km}^{2}$. La population de la Région est estimée au dernier recensement de 2010 au Togo à 837437 habitants (D.G.S.C.N.T, 2011). Les langues locales les plus parlées sont l'Ewé et le Mina, le Français étant la langue officielle. Le relief de la zone est caractérisé par le Bassin sédimentaire côtier de la Région Maritime constitué de sable, de calcaire et d'argile essentiellement. C'est une vaste plaine aux vallées relativement basses et plates qui constituent une véritable cuvette où coulent de nombreuses rivières. Le climat est de type subtropical avec deux saisons de pluies alternées de deux saisons sèches. Ce climat est à dominance tropicale avec une Mousson caractérisée par des pluies moins importantes et de l'harmattan avec des précipitations annuelles moyennes de $859 \mathrm{~mm}$. La température moyenne à Lomé est de $26.8{ }^{\circ} \mathrm{C}$ (Koudouvo, 2009). 


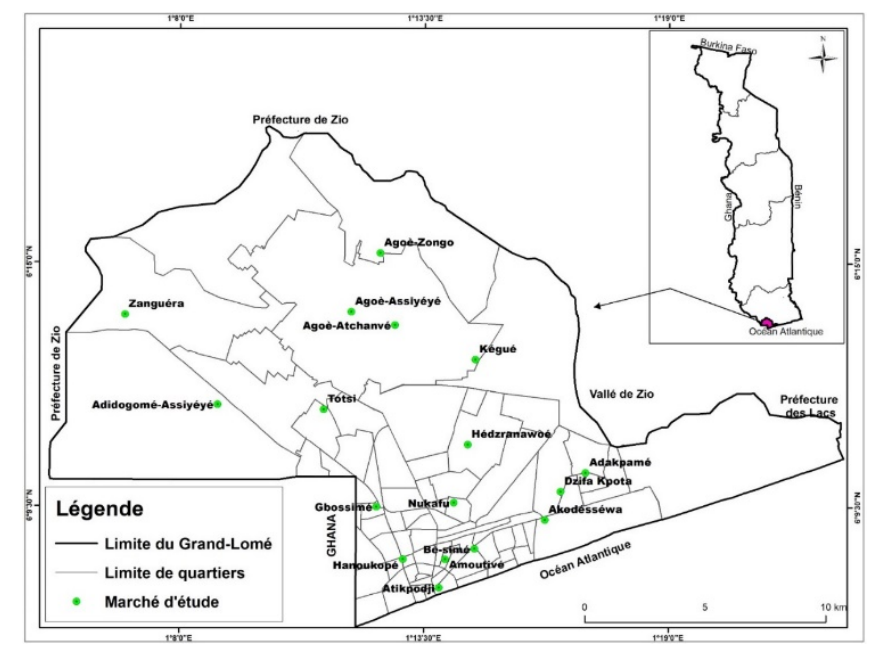

Figure 1. Carte représentant les marchés de vente de la Zone d'étude (Région Sanitaire Lomé-Commune du Togo)

Source:Koudouvo et al., 2011

\section{Collecte des données}

De Mai 2018 à Juillet 2019, 34 herboristes de marché répartis dans 17 marchés de la RSLC à raison de 2 par marché, ont été enquêtés en utilisant la méthode d'enquête ethnobotanique ATRM (Achat en Triplet de Recettes Médicinales) (Koudouvo, 2009, Koudouvo et al., 2016) pour la collecte des données. La méthode consiste à aller trois fois de suite dans l'intervalle de deux semaines acheter de recettes auprès d'un même herboriste de marché pour soigner une même maladie. Les recettes qui seront achetées lors de ces trois visites chez chaque herboriste, sont respectivement en grand nombre (GN), en nombre réduit (NR), et en nombre très réduit (TR). Trente et quatre herboristes dans 17 marchés de la zone d'études ont été visités pour les achats. Les informations recueillies sur ces recettes ont portées sur les caractéristiques ethnobotaniques et ethnopharmacologiques telles que le mode de préparation, les voies d'administration, le mode d'emploi, la posologie, les interdits alimentaires lors de leur utilisation, les effets secondaires et indésirables éventuels.

\section{Traitement et analyse des données}

La flore Analytique du Bénin (Akoègninou et al., 2006) et la base de données en ligne de PROTA4U (Plant Resources of Tropical Africa) : www.prota4u.org , ont été utilisées pour l'identification botanique des espèces de plantes qui composent les recettes recensées. Les identifications ethnobotaniques qui ont été réalisées en suivant les travaux de Koudouvo et al., (2016), ont été confirmées par l'équipe du Prof. Koffi Akpagana du Togo. La précision des noms en langues locales a été réalisée en référence à 
Akoègninou et al., (2006), à Adjanohoun et al., (1986, 1989), au Dictionnaire Monographique de l'Afrique de l'Ouest (Eklu-Natey et al., 2012) et à la Pharmacopée de l'Afrique de l'Ouest, Edition1 (PAO1) de 2013. Des logiciels ethnobotaniques récents ont servi aux traitements des données recueillies. La Sélection par Elimination Progressive (SEP) (Koudouvo et al., 2016, Denou et al., 2016 ; Koudouvo, 2009) reprise par Assouma et al., (2018) en Sélection par Elimination Progressive Améliorée (SEPA), a permis la sélection de plantes pour de futurs tests de laboratoire.

La détermination de la Valeur d'usuelle (VU), une méthode quantitative pour évaluer l'importance relative des espèces connues localement, a été calculée selon Koudouvo (2009) ; Aburjai et al., (2006) ; Gazzaneo et al., (2005), suivant la formule :

$\mathrm{UV}=\Sigma \mathrm{U} / \mathbf{n}$ où $\mathbf{U}$ est le nombre de citations par espèce et $\mathbf{n}$ le nombre d'Herboristes enquêtés.

La corrélation entre la proportion des organes renouvelables et non renouvelables comme parties utilisées des plantes sur les marchés (Koudouvo et al., 2017), permettra de statuer sur la bonne pratique en cueillette de plantes médicinales pour la préservation des espèces contre la menace de la biodiversité.

\section{Résultats}

\section{Caractéristiques botaniques et diversités biologiques des espèces}

La présente étude a permis de recenser 102 recettes de plantes médicinales auprès des 34 herboristes de marchés qui sont exclusivement des femmes. Ces recettes sont constituées par 68 espèces appartenant à 38 familles botaniques dont les plus représentées sont les Annonaceae et Leguminosae-Caesalpinioideae (5 espèces chacune), Apocynaceae, Euphorbiaceae, et Leguminosae-Papilionoideae (4 espèces chacune). Anacardiaceae (129 citations), Euphorbiaceae (75 citations), et LeguminosaeMimosoideae (63 citations), ont été les familles pour lesquelles les espèces ont étés les plus citées (Figure 2). Du point de vue fréquence $(\mathrm{F})$ des espèces, Lannea kerstingii ( $\mathrm{F}=9,62 \%)$, Bridelia ferruginea ( $\mathrm{F}=8,39 \%)$, et Zanthoxylum xanthoxyloides $(\mathrm{F}=7,65 \%)$ ) ont été les espèces les plus fréquemment citées (Tableau 1). Les types morphologiques d'appartenance de ces espèces (Figure 3 ) sont entre autres les arbres (39,71\%), les herbes (38,24\%), les arbustes (14,7\%), les lianes (2,94\%), les arbrisseaux (2,94\%), et les palmiers $(1,47 \%)$. 


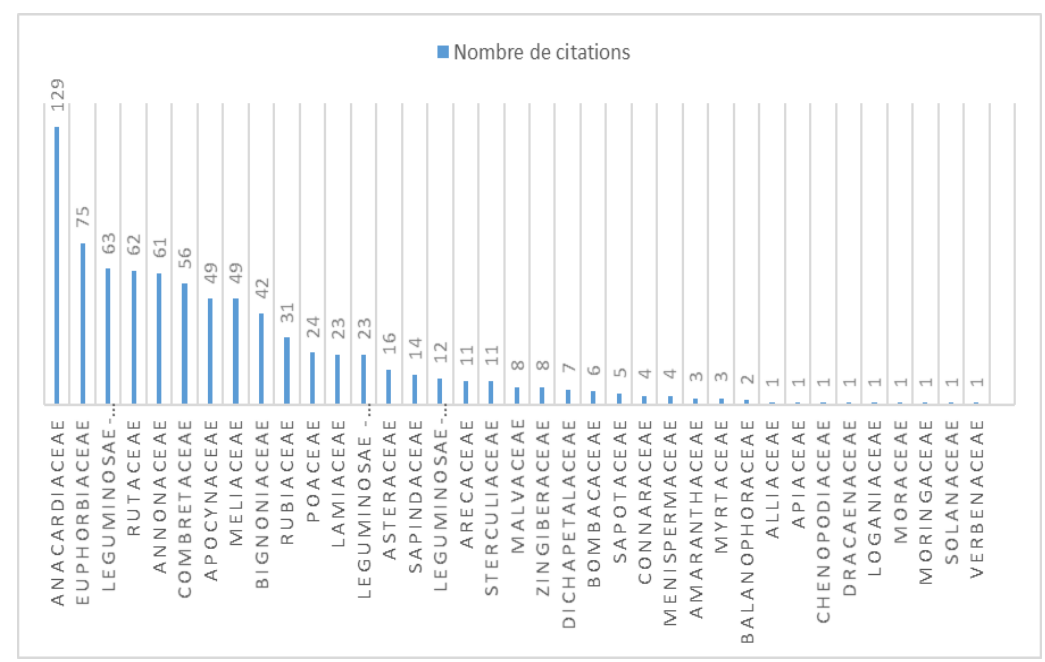

Figure 2. Fréquence de citation des espèces par famille

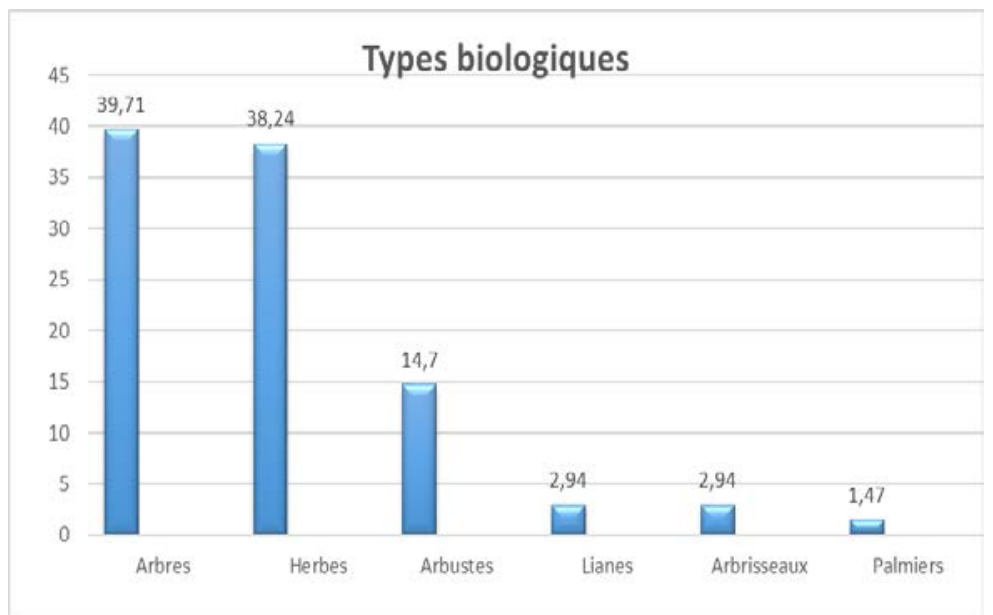

Figure 3. Histogramme par types morphologiques

\section{Caractéristiques ethnopharmacologiques des plantes et recettes de plantes}

Tableau 1. Diversité biologique des espèces anti-infectieuses de la Région Sanitaire "Lomé-Commune" du Togo

\begin{tabular}{|c|c|c|c|c|c|c|c|}
\hline $\begin{array}{c}\text { Noms } \\
\text { scientifiques }\end{array}$ & Familles & $\begin{array}{c}\text { Noms en } \\
\text { langues } \\
\text { locales } \\
\text { (Langues) }\end{array}$ & $\begin{array}{c}\text { Types } \\
\text { morphologiques }\end{array}$ & $\begin{array}{c}\text { Parties } \\
\text { utilisées }\end{array}$ & \multicolumn{2}{|c|}{$\begin{array}{c}\text { Citation des } \\
\text { espèces }\end{array}$} & $\begin{array}{c}\text { Valeurs } \\
\text { usuelles }\end{array}$ \\
\cline { 3 - 8 } & & Nombr & $\begin{array}{c}\text { Fréque } \\
\text { nce(\%) }\end{array}$ & \\
\hline $\begin{array}{c}\text { Lannea } \\
\text { kerstingii A. } \\
\text { Rich. }\end{array}$ & $\begin{array}{c}\text { Anacardi } \\
\text { aceae }\end{array}$ & $\begin{array}{c}\text { Melonkou }= \\
\text { Melankou }= \\
\text { Monlonkou }\end{array}$ & Arbre & ET & 78 & $9,62 \%$ & 2,29 \\
\hline $\begin{array}{c}\text { Bridelia } \\
\text { ferruginea } \\
\text { Benth. }\end{array}$ & $\begin{array}{c}\text { Euphorbi } \\
\text { aceae }\end{array}$ & $\begin{array}{c}\text { Kamati }= \\
\text { Hlihoin }\end{array}$ & Arbuste & ET & 68 & $8,39 \%$ & 2,00 \\
\hline
\end{tabular}




\begin{tabular}{|c|c|c|c|c|c|c|c|}
\hline $\begin{array}{c}\text { Zanthoxylum } \\
\text { xanthoxyloide } \\
\text { s Lam. }\end{array}$ & Rutaceae & $\begin{array}{l}\text { Exeti = Exe } \\
\quad=\mathrm{Xe}\end{array}$ & Arbuste & $\mathrm{Ra}$ & 62 & $7,65 \%$ & 1,82 \\
\hline $\begin{array}{c}\text { Pteleopsis } \\
\text { suberosa } \\
\text { Engl. \& Diels. }\end{array}$ & $\begin{array}{c}\text { Combret } \\
\text { aceae }\end{array}$ & $\begin{array}{c}\text { Kotokolikan } \\
=\text { Sissinon = } \\
\text { Aklitsi }\end{array}$ & Arbre & ET & 56 & $6,91 \%$ & 1,64 \\
\hline $\begin{array}{c}\text { Mangifera } \\
\text { indica Linn. }\end{array}$ & $\begin{array}{c}\text { Anacardi } \\
\text { aceae }\end{array}$ & $\begin{array}{c}\text { Mangoti = } \\
\text { Atontsi }\end{array}$ & Arbre & ET & 51 & $6,29 \%$ & 1,50 \\
\hline $\begin{array}{c}\text { Khaya } \\
\text { senegalensis } \\
\text { (Desr.) A. } \\
\text { Juss. }\end{array}$ & $\begin{array}{l}\text { Meliacea } \\
\text { e }\end{array}$ & $\begin{array}{c}\text { Mahogen = } \\
\text { Mahogani }\end{array}$ & Arbre & ET & 49 & $6,04 \%$ & 1,44 \\
\hline $\begin{array}{c}\text { Uvaria } \\
\text { chamae P. } \\
\text { Beauv. }\end{array}$ & $\begin{array}{c}\text { Annonac } \\
\text { eae }\end{array}$ & $\begin{array}{c}\text { Agbanlan = } \\
\text { Gbana- } \\
\text { gbana }\end{array}$ & Arbuste & $\mathrm{Ra}$ & 49 & $6,04 \%$ & 1,44 \\
\hline $\begin{array}{l}\text { Alstonia } \\
\text { boonei De } \\
\text { Wild. }\end{array}$ & $\begin{array}{l}\text { Apocyna } \\
\text { ceae }\end{array}$ & $\begin{array}{c}\text { Nyami Dua } \\
=\text { Nyamidua } \\
=\text { Ton-Ton }= \\
\text { Siaketekre }\end{array}$ & Arbre & ET & 43 & $5,30 \%$ & 1,26 \\
\hline $\begin{array}{c}\text { Parkia } \\
\text { biglobosa } \\
\text { (Jacq.) Benth }\end{array}$ & $\begin{array}{l}\text { Legumin } \\
\text { osae- } \\
\text { Mimosoi } \\
\text { deae } \\
\end{array}$ & $\begin{array}{c}\text { Wokpa }=\text { Wot } \\
\text { si }\end{array}$ & Arbre & ET & 29 & $3,58 \%$ & 0,85 \\
\hline $\begin{array}{c}\underline{\text { Acacia }} \\
\frac{\text { nilotica }(\mathrm{L} .)}{\text { Willd. ex }} \\
\text { Delile }\end{array}$ & $\begin{array}{l}\text { Legumin } \\
\text { osae- } \\
\text { Mimosoi } \\
\text { deae }\end{array}$ & Ayigbé & Arbre & Fr & 26 & $3,20 \%$ & 0,76 \\
\hline $\begin{array}{c}\text { Kigelia } \\
\text { africana } \\
\text { (Lam.) Benth. }\end{array}$ & $\begin{array}{c}\text { Bignonia } \\
\text { ceae }\end{array}$ & $\begin{array}{l}\text { Vinokpa = } \\
\text { Gnakpekpe }\end{array}$ & Arbre & ET & 26 & $3,20 \%$ & 0,76 \\
\hline $\begin{array}{l}\text { Acanthosperm } \\
\text { um hispidum } \\
\text { (DC). }\end{array}$ & $\begin{array}{c}\text { Asterace } \\
\text { ae }\end{array}$ & $\begin{array}{c}\text { Afegban = } \\
\text { Dougban = } \\
\text { Afegbin = } \\
\text { Damelatsu'n } \\
\text { ko }\end{array}$ & Herbe & $\mathrm{PE}$ & 16 & $1,97 \%$ & 0,47 \\
\hline $\begin{array}{c}\text { Spathodea } \\
\text { campanulata } \\
\text { P. Beauv. }\end{array}$ & $\begin{array}{l}\text { Bignonia } \\
\text { ceae }\end{array}$ & $\begin{array}{l}\text { Adassigolo } \\
=\text { Adassigo } \\
=\text { Adatigolo } \\
=\text { Adatsigo }\end{array}$ & Arbre & ET & 16 & $1,97 \%$ & 0,47 \\
\hline $\begin{array}{c}\text { Ocimum } \\
\text { gratissimum } \\
\text { Linn. }\end{array}$ & $\begin{array}{c}\text { Lamiace } \\
\text { ae }\end{array}$ & $\begin{array}{l}\text { Esroun = } \\
\text { Dzogbeti }\end{array}$ & Herbe & $\mathrm{TF}$ & 15 & $1,85 \%$ & 0,44 \\
\hline $\begin{array}{c}\text { Paullinia } \\
\text { pinnata Linn. }\end{array}$ & $\begin{array}{l}\text { Sapindac } \\
\text { eae }\end{array}$ & $\begin{array}{c}\text { Assiviatoè= } \\
\text { Assiviatoèka } \\
=\text { Hokouika } \\
= \\
\text { Gbexatsaxat } \\
\text { sa } \\
\end{array}$ & Liane & $\mathrm{TF}$ & 14 & $1,72 \%$ & 0,41 \\
\hline
\end{tabular}




\begin{tabular}{|c|c|c|c|c|c|c|c|}
\hline $\begin{array}{c}\text { Sarcocephalus } \\
\text { latifolius(Sm.) } \\
\text { E.A.Bruce }\end{array}$ & $\begin{array}{c}\text { Rubiacea } \\
\text { e }\end{array}$ & Nimon & Herbe & $\mathrm{Ra}$ & 13 & $1,60 \%$ & 0,38 \\
\hline $\begin{array}{c}\text { Cocos } \\
\text { nucifera Linn. }\end{array}$ & $\begin{array}{c}\text { Arecacea } \\
\text { e }\end{array}$ & $\begin{array}{c}\text { Yovoninti }= \\
\text { Yovonèti = } \\
\text { Yevunetsi = } \\
\text { Netsi }\end{array}$ & Palmier & $\mathrm{Ra}$ & 11 & $1,35 \%$ & 0,32 \\
\hline $\begin{array}{l}\text { Oldenlandia } \\
\text { corymbosa } \\
\text { Linn. }\end{array}$ & $\begin{array}{c}\text { Rubiacea } \\
\text { e }\end{array}$ & $\begin{array}{c}\text { Tsoèvissihin } \\
= \\
\text { Vouvoudralè }\end{array}$ & Herbe & $\mathrm{TF}$ & 10 & $1,23 \%$ & 0,29 \\
\hline $\begin{array}{c}\text { Cassia } \\
\text { sieberiana } \\
\text { DC. }\end{array}$ & $\begin{array}{c}\text { Legumin } \\
\text { osae - } \\
\text { Caesalpi } \\
\text { nioideae }\end{array}$ & Gati gati & Arbuste & $\mathrm{Ra}$ & 9 & $1,11 \%$ & 0,26 \\
\hline $\begin{array}{c}\text { Indigofera } \\
\text { pulchra Willd. }\end{array}$ & $\begin{array}{l}\text { Legumin } \\
\text { osae- } \\
\text { Papiliono } \\
\text { ideae } \\
\end{array}$ & $\begin{array}{c}\text { Dzokon = } \\
\text { Zokon = } \\
\text { Zoukon }\end{array}$ & Herbe & $\mathrm{PE} / \mathrm{Fe}$ & 9 & $1,11 \%$ & 0,26 \\
\hline $\begin{array}{c}\text { Piliostigma } \\
\text { thonningii } \\
\text { (Sch.) Miln. } \\
\text { Redh. }\end{array}$ & $\begin{array}{c}\text { Legumin } \\
\text { osae - } \\
\text { Caesalpi } \\
\text { nioideae }\end{array}$ & $\begin{array}{c}\text { Klonti = } \\
\text { Eklonti = } \\
\text { Eklo = Kloè }\end{array}$ & Arbre & $\mathrm{ET} / \mathrm{Fe}$ & 9 & $1,11 \%$ & 0,26 \\
\hline $\begin{array}{c}\text { Vetiveria } \\
\text { nigritana } \\
\text { (Benth.) Stapf }\end{array}$ & Poaceae & $\begin{array}{c}\text { Kédéké= } \\
\text { Kéréké = } \\
\text { Kédéka = } \\
\text { Gbé woyè } \\
\text { woyè = } \\
\text { Gbéwoayé }\end{array}$ & Herbe & $\mathrm{Ra}$ & 9 & $1,11 \%$ & 0,26 \\
\hline $\begin{array}{c}\text { Xylopia } \\
\text { aethiopica } \\
\text { (Dunal) A. } \\
\text { Rich. }\end{array}$ & $\begin{array}{c}\text { Annonac } \\
\text { eae }\end{array}$ & Etso $=$ Esso & Arbre & Fr & 9 & $1,11 \%$ & 0,26 \\
\hline $\begin{array}{l}\text { Cymbopogon } \\
\text { citratus Stapf. }\end{array}$ & Poaceae & $\begin{array}{l}\text { Tsigbe = } \\
\text { Gbehoin }\end{array}$ & Herbe & $\mathrm{TF}$ & 8 & $0,92 \%$ & 0,23 \\
\hline $\begin{array}{c}\text { Ocimum } \\
\text { canum Sams }\end{array}$ & $\begin{array}{c}\text { Lamiace } \\
\text { ae }\end{array}$ & $\begin{array}{c}\text { Ahamè = } \\
\text { Hamè }\end{array}$ & Herbe & $\mathrm{PE}$ & 8 & $0,92 \%$ & 0,23 \\
\hline $\begin{array}{c}\text { Tetrapleura } \\
\text { tetraptera (Sc } \\
\text { hum. \& } \\
\text { Thonn.) Taub }\end{array}$ & $\begin{array}{l}\text { Legumin } \\
\text { osae- } \\
\text { Mimosoi } \\
\text { deae } \\
\end{array}$ & Plèkèssè & Arbre & Fr & 8 & $0,92 \%$ & 0,23 \\
\hline $\begin{array}{c}\text { Dichapetalum } \\
\text { madagascarie } \\
\text { nse (DC) } \\
\text { Keay. }\end{array}$ & $\begin{array}{c}\text { Dichapet } \\
\text { alaceae }\end{array}$ & $\begin{array}{c}\text { Atihali }= \\
\text { Atihanli = } \\
\text { Atixali }= \\
\text { Tchokpleti }\end{array}$ & Herbe & Fr & 7 & $0,86 \%$ & 0,20 \\
\hline $\begin{array}{c}\text { Gossypium } \\
\text { hirsutum Linn. }\end{array}$ & $\begin{array}{c}\text { Malvace } \\
\text { ae }\end{array}$ & $\begin{array}{c}\text { Deti }=\text { Edeti } \\
=\text { Detitsi }= \\
\text { Detifutsi }\end{array}$ & Herbe & $\mathrm{TF}$ & 7 & $0,86 \%$ & 0,20 \\
\hline $\begin{array}{c}\frac{\text { Sorghum }}{\text { bicolor (Linn.) }} \\
\underline{\text { Moench }}\end{array}$ & Poaceae & $\begin{array}{c}\text { Adako = } \\
\text { Ada }= \\
\text { Adadzen }\end{array}$ & Herbe & $\mathrm{Fe}$ & 7 & $0,86 \%$ & 0,20 \\
\hline
\end{tabular}




\begin{tabular}{|c|c|c|c|c|c|c|c|}
\hline $\begin{array}{c}\text { Aframomum } \\
\text { melegueta } \\
\text { K.Schum. }\end{array}$ & $\begin{array}{l}\text { Zinginbe } \\
\text { raceae }\end{array}$ & Atakou & Herbe & $\mathrm{Ra} / \mathrm{Fr}$ & 6 & $0,74 \%$ & 0,17 \\
\hline $\begin{array}{c}\text { Waltheria } \\
\text { indica Linn. }\end{array}$ & $\begin{array}{l}\text { Sterculia } \\
\text { ceae }\end{array}$ & $\begin{array}{c}\text { Adouwèti } \\
=\text { Sinto = } \\
\text { Sin-to = } \\
\text { Adoufanti = } \\
\text { Seto }\end{array}$ & Herbe & $\mathrm{TF}$ & 6 & $0,74 \%$ & 0,17 \\
\hline $\begin{array}{c}\text { Ceiba } \\
\text { pentandra (L.) }\end{array}$ & $\begin{array}{c}\text { Bombaca } \\
\text { ceae }\end{array}$ & Hounti & Arbre & ET & 5 & $0,61 \%$ & 0,14 \\
\hline $\begin{array}{l}\text { Cola millenii } \\
\text { K. Schum. }\end{array}$ & $\begin{array}{l}\text { Sterculia } \\
\text { ceae }\end{array}$ & $\begin{array}{c}\text { Assiviatoe = } \\
\text { Kessedui = } \\
\text { Kesseloin = } \\
\text { Kpando }\end{array}$ & Arbre & TF/Ra & 5 & $0,61 \%$ & 0,14 \\
\hline $\begin{array}{c}\text { Vitellaria } \\
\text { paradoxa } \\
\text { Gaertn C.F } \\
\end{array}$ & $\begin{array}{c}\text { Sapotace } \\
\text { ae }\end{array}$ & Yokpa & Arbre & ET & 5 & $0,61 \%$ & 0,14 \\
\hline $\begin{array}{c}\text { Rourea } \\
\text { coccinea } \\
\text { (Thonn. ex } \\
\text { Sch.) Benth. }\end{array}$ & $\begin{array}{c}\text { Connarac } \\
\text { eae }\end{array}$ & $\begin{array}{l}\text { Tomegavi }= \\
\text { Hlongbedeti }\end{array}$ & Arbuste & $\mathrm{Ra}$ & 4 & $0,49 \%$ & 0,11 \\
\hline $\begin{array}{l}\text { Euphorbia } \\
\text { hirta Linn. }\end{array}$ & $\begin{array}{l}\text { Euphorbi } \\
\text { aceae }\end{array}$ & $\begin{array}{c}\text { Anonsikan = } \\
\text { Nosikin = } \\
\text { Notsika = } \\
\text { Anotsigbe }\end{array}$ & Herbe & $\mathrm{PE}$ & 4 & $0,49 \%$ & 0,11 \\
\hline $\begin{array}{c}\text { Morinda } \\
\text { lucida Linn }\end{array}$ & $\begin{array}{c}\text { Rubiacea } \\
\text { e }\end{array}$ & $\begin{array}{c}\text { Zanklan = } \\
\text { Dadaklan = } \\
\text { Dzadzaklan }\end{array}$ & Arbre & $\mathrm{Ra}$ & 4 & $0,49 \%$ & 0,11 \\
\hline $\begin{array}{c}\text { Pavetta } \\
\text { corymbosa } \\
\text { (DC) F.N. } \\
\text { Will. }\end{array}$ & $\begin{array}{c}\text { Rubiacea } \\
\text { e }\end{array}$ & $\begin{array}{c}\text { Sifafa }= \\
\text { Siafa }= \\
\text { Siafati }\end{array}$ & Arbuste & $\mathrm{PE}$ & 4 & $0,49 \%$ & 0,11 \\
\hline $\begin{array}{l}\text { Pleiocarpa } \\
\text { pycnantha } \\
\text { (K. Sch.) } \\
\text { Stapf. }\end{array}$ & $\begin{array}{c}\text { Apocyna } \\
\text { ceae }\end{array}$ & $\begin{array}{l}\text { Avlokouigbe } \\
=\text { Gbevenati }\end{array}$ & Arbuste & $\mathrm{TF}$ & 4 & $0,49 \%$ & 0,11 \\
\hline $\begin{array}{c}\text { Tiliacora } \\
\text { funifera Oliv. }\end{array}$ & $\begin{array}{c}\text { Menisper } \\
\text { maceae }\end{array}$ & Katokpan & Liane & $\mathrm{Ra}$ & 4 & $0,49 \%$ & 0,11 \\
\hline $\begin{array}{c}\text { Eugenia caryo } \\
\text { phyllata Thun } \\
\text { b }\end{array}$ & $\begin{array}{c}\text { Myrtacea } \\
\text { e }\end{array}$ & $\begin{array}{l}\text { Plingota }= \\
\text { Plèplè }\end{array}$ & Arbre & $\mathrm{Fr}$ & 3 & $0,37 \%$ & 0,08 \\
\hline $\begin{array}{c}\text { Gomphrena } \\
\text { celosioides } \\
\text { Mart. }\end{array}$ & $\begin{array}{c}\text { Amarant } \\
\text { haceae }\end{array}$ & $\begin{array}{c}\text { Papataxe = } \\
\text { Gnagantaxe } \\
= \\
\text { Gnagantahé }\end{array}$ & Herbe & $\mathrm{PE}$ & 3 & $0,37 \%$ & 0,08 \\
\hline $\begin{array}{l}\text { Chamaecrista } \\
\text { rotundifolia } \\
\text { (Pers.) }\end{array}$ & $\begin{array}{c}\text { Legumin } \\
\text { osae - } \\
\text { Caesalpi } \\
\text { nioideae }\end{array}$ & $\begin{array}{c}\text { Azingbe = } \\
\text { Zingbe }= \\
\text { Zigbe }\end{array}$ & Herbe & $\mathrm{TF}$ & 2 & $0,24 \%$ & 0,05 \\
\hline
\end{tabular}




\begin{tabular}{|c|c|c|c|c|c|c|c|}
\hline $\begin{array}{c}\text { Phyllanthus } \\
\text { amarus Sch. et } \\
\text { Th. }\end{array}$ & $\begin{array}{l}\text { Euphorbi } \\
\text { aceae }\end{array}$ & $\begin{array}{c}\text { Ehlinvi = } \\
\text { Hlinvi }\end{array}$ & Herbe & $\mathrm{PE}$ & 2 & $0,24 \%$ & 0,05 \\
\hline $\begin{array}{l}\text { Tamarindus } \\
\text { indica Linn. }\end{array}$ & $\begin{array}{l}\text { Legumin } \\
\text { osae - } \\
\text { Caesalpi } \\
\text { nioideae }\end{array}$ & $\begin{array}{l}\text { Atikessigbe } \\
=\text { Kététiya }\end{array}$ & Arbre & $\mathrm{Fr}$ & 2 & $0,24 \%$ & 0,05 \\
\hline $\begin{array}{c}\text { Thonningia } \\
\text { sanguinea } \\
\text { Vahl. }\end{array}$ & $\begin{array}{c}\text { Balanoph } \\
\text { oraceae }\end{array}$ & $\begin{array}{l}\text { Pidè }= \\
\text { Pide }\end{array}$ & Herbe & $\mathrm{Ra}$ & 2 & $0,24 \%$ & 0,05 \\
\hline $\begin{array}{c}\text { Zingiber } \\
\text { officinale } \\
\text { Rosc. Trans. } \\
\text { Linn. }\end{array}$ & $\begin{array}{c}\text { Zingiber } \\
\text { aceae }\end{array}$ & Dotè & Herbe & $\mathrm{Rh}$ & 2 & $0,24 \%$ & 0,05 \\
\hline $\begin{array}{c}\text { Abrus } \\
\text { precatorius L. }\end{array}$ & $\begin{array}{l}\text { Legumin } \\
\text { osae- } \\
\text { Papiliono } \\
\text { ideae } \\
\end{array}$ & $\begin{array}{c}\text { Djenkoudjen } \\
= \\
\text { Djindjinkou } \\
\text { djin }\end{array}$ & Arbuste & $\mathrm{Ra}$ & 1 & $0,12 \%$ & 0,02 \\
\hline $\begin{array}{c}\text { Adansonia } \\
\text { digitata Linn. }\end{array}$ & $\begin{array}{c}\text { Bombaca } \\
\text { ceae }\end{array}$ & $\begin{array}{c}\text { Adidotsi = } \\
\text { Lagbati = } \\
\text { Lagbadido }\end{array}$ & Arbre & ET & 1 & $0,12 \%$ & 0,02 \\
\hline $\begin{array}{c}\text { Allium } \\
\text { sativum L, }\end{array}$ & Alliaceae & Ayo & Herbe & $\mathrm{Bu}$ & 1 & $0,12 \%$ & 0,02 \\
\hline $\begin{array}{c}\text { Annona } \\
\text { senegalensis } \\
\text { Pers. }\end{array}$ & $\begin{array}{c}\text { Annonac } \\
\text { eae }\end{array}$ & Zogbényigli & Arbuste & $\mathrm{TF}$ & 1 & $0,12 \%$ & 0,02 \\
\hline $\begin{array}{c}\text { Annona } \\
\text { squamosa } \mathrm{L} .\end{array}$ & $\begin{array}{c}\text { Annonac } \\
\text { eae }\end{array}$ & $\begin{array}{l}\text { Kandrouwa } \\
=\text { Cannelle } \\
\text { africaine } \\
\end{array}$ & Arbuste & ET & 1 & $0,12 \%$ & 0,02 \\
\hline $\begin{array}{c}\text { Anthocleista } \\
\text { nobilis/A. } \\
\text { djalonensis A. } \\
\text { Cher. }\end{array}$ & $\begin{array}{c}\text { Loganiac } \\
\text { eae }\end{array}$ & Gboloba & Arbre & $\mathrm{Ra}$ & 1 & $0,12 \%$ & 0,02 \\
\hline $\begin{array}{c}\text { Caesalpinia } \\
\text { bonduc Roxb. }\end{array}$ & $\begin{array}{c}\text { Legumin } \\
\text { osae - } \\
\text { Caesalpi } \\
\text { nioideae }\end{array}$ & $\begin{array}{c}\text { Adikou = } \\
\text { Adikutsi }\end{array}$ & Arbrisseau & $\mathrm{Fe}$ & 1 & $0,12 \%$ & 0,02 \\
\hline $\begin{array}{l}\text { Capsicum } \\
\text { annuun } L,\end{array}$ & $\begin{array}{c}\text { Solanace } \\
\text { ae }\end{array}$ & Yébéssévi & Herbe & Fr & 1 & $0,12 \%$ & 0,02 \\
\hline $\begin{array}{c}\text { Chenopodium } \\
\text { ambrosioides } \\
\text { Linn. }\end{array}$ & $\begin{array}{c}\text { Chenopo } \\
\text { diaceae }\end{array}$ & $\begin{array}{l}\text { Magbezondé } \\
=\text { Azondé = } \\
\text { Magbezonli }\end{array}$ & Herbe & $\mathrm{TF}$ & 1 & 0,12 & 0,02 \\
\hline $\begin{array}{c}\text { Desmodium } \\
\text { tortuosum } \\
\text { (Sw.) DC }\end{array}$ & $\begin{array}{l}\text { Legumin } \\
\text { osae- } \\
\text { Papiliono } \\
\text { ideae }\end{array}$ & $\begin{array}{c}\text { Tedavonou } \\
= \\
\text { Telanonnou } \\
\quad= \\
\text { Tedeavonou }\end{array}$ & Herbe & $\mathrm{Fe}$ & 1 & $0,12 \%$ & 0,02 \\
\hline $\begin{array}{c}\text { Ficus } \\
\text { platyphylla } \\
\text { Del } \\
\end{array}$ & $\begin{array}{c}\text { Moracea } \\
\text { e }\end{array}$ & $\begin{array}{c}\text { Vodzin = } \\
\text { Vodzentsi }\end{array}$ & Arbre & $\mathrm{TF}$ & 1 & $0,12 \%$ & 0,02 \\
\hline
\end{tabular}




\begin{tabular}{|c|c|c|c|c|c|c|c|}
\hline $\begin{array}{c}\text { Hibiscus } \\
\text { surattensis L }\end{array}$ & $\begin{array}{c}\text { Malvace } \\
\text { ae }\end{array}$ & $\begin{array}{c}\text { Gnatoxe = } \\
\text { Gnatu }\end{array}$ & Herbe & $\mathrm{TF}$ & 1 & $0,12 \%$ & 0,02 \\
\hline $\begin{array}{l}\text { Holarrhena } \\
\text { floribunda (G. } \\
\text { Don) Dw. } \\
\text { Sch. }\end{array}$ & $\begin{array}{l}\text { Apocyna } \\
\text { ceae }\end{array}$ & $\begin{array}{c}\text { Sessewou }= \\
\text { Sewu }\end{array}$ & Arbre & $\mathrm{Ra}$ & 1 & $0,12 \%$ & 0,02 \\
\hline $\begin{array}{c}\text { Jatropha } \\
\text { gossypifolia } \\
\text { Linn. }\end{array}$ & $\begin{array}{l}\text { Euphorbi } \\
\text { aceae }\end{array}$ & $\begin{array}{c}\text { Babatidjin = } \\
\text { Babatidjê = } \\
\text { Kpoti }\end{array}$ & Arbuste & $\mathrm{TF}$ & 1 & $0,12 \%$ & 0,02 \\
\hline $\begin{array}{c}\text { Lippia } \\
\text { multiflora } \\
\text { Moldenke }\end{array}$ & $\begin{array}{c}\text { Verbenac } \\
\text { eae }\end{array}$ & $\begin{array}{l}\text { Avudati }= \\
\text { Avloti }\end{array}$ & Arbre & $\mathrm{TF}$ & 1 & $0,12 \%$ & 0,02 \\
\hline $\begin{array}{c}\text { Lonchocarpus } \\
\text { sericeus } \\
\text { (Poir.) Kunth }\end{array}$ & $\begin{array}{l}\text { Legumin } \\
\text { osae- } \\
\text { Papiliono } \\
\text { ideae }\end{array}$ & Lamba & Arbre & ET & 1 & $0,12 \%$ & 0,02 \\
\hline $\begin{array}{c}\text { Monodora } \\
\text { myristica } \\
\text { (Gaertn) }\end{array}$ & $\begin{array}{c}\text { Annonac } \\
\text { eae }\end{array}$ & Ayikou & Arbre & Fr & 1 & $0,12 \%$ & 0,02 \\
\hline $\begin{array}{c}\text { Moringa } \\
\text { oleifera Linn. }\end{array}$ & $\begin{array}{l}\text { Moringa } \\
\text { ceae }\end{array}$ & $\begin{array}{c}\text { Yovovigbe }= \\
\text { Kpotsi }\end{array}$ & Arbre & $\mathrm{Ra}$ & 1 & $0,12 \%$ & 0,02 \\
\hline $\begin{array}{c}\text { Pimpinella } \\
\text { anisum L }\end{array}$ & Apiacées & Pastis ku & Herbe & Fr & 1 & $0,12 \%$ & 0,02 \\
\hline $\begin{array}{l}\text { Rauvolfia } \\
\text { vomitoria } \\
\text { Afzel. }\end{array}$ & $\begin{array}{l}\text { Apocyna } \\
\text { ceae }\end{array}$ & $\begin{array}{c}\text { Dodémakpo } \\
\text { woe }\end{array}$ & Arbrisseau & $\mathrm{Ra}$ & 1 & $0,12 \%$ & 0,02 \\
\hline $\begin{array}{c}\text { Sanseviera } \\
\text { liberica Ger. } \\
\text { and Labr. }\end{array}$ & $\begin{array}{c}\text { Dracaena } \\
\text { ceae }\end{array}$ & $\begin{array}{c}\text { Yodobou = } \\
\text { Yodobo }= \\
\text { Yoboo }\end{array}$ & Herbe & $\mathrm{Rh}$ & 1 & $0,12 \%$ & 0,02 \\
\hline
\end{tabular}

Note $: \mathrm{ER}=$ Ecorce de Racine, $\mathrm{ET}=$ Ecorce de Tige, $\mathrm{Bu}=$ Bulbe, $\mathrm{Fe}=$ Feuille, $\mathrm{Fr}=$

Fruit, $\mathrm{Gr}=$ Graine, $\mathrm{PE}=$ Plante Entière, $\mathrm{Ra}=$ Racine, $\mathrm{TF}=$ Tige Feuillée, $\mathrm{Rh}=$ Rhizome

\section{Organes utilisés, modes de préparation et voies d'administration}

Ecorce de tronc $(53,57 \%)$, racine (16,13\%), tige feuillées $(6,90 \%)$, plante entière (6,65\%), fruit (6,28\%), et écorce de racine (6,04\%) ont été les parties les plus utilisées des espèces recensées (Figure 4). Les autres parties utilisées sont les feuilles (3,57\%), rhizome $(0,49 \%)$, graine $(0,25 \%)$ et bulbe $(0,12 \%)$. Le prélèvement des organes non renouvelables (écorce de tronc, écorce racine, racine, rhizome, plante entière et bulbe) représente plus de 83\% alors que celui des organes renouvelables (fruit, tige feuillée, feuille et graine) n'est qu'à moins de $17 \%$, ce qui constitue une véritable menace de la biodiversité médicinale. 
Ces espèces sont préparées en décoction (93.14\%) et en macération (6,86\%) (Figure 5) puis administrées en exclusivité par voie orale $(100 \%)$, pour soigner les maladies infectieuses.

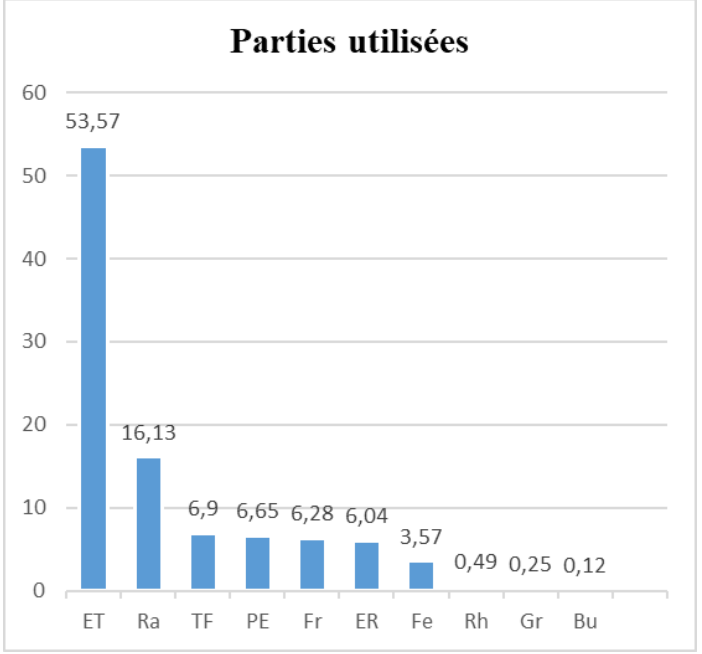

$$
\begin{gathered}
\text { ER = Ecorce de } \\
\text { Racine } \\
\text { ET = Ecorce de Tige } \\
\text { Bu = Bulbe } \\
\text { Fe }=\text { Feuille } \\
\text { Fr }=\text { Fruit } \\
\text { Gr }=\text { Graine } \\
\text { PE = Plante Entière } \\
\text { Ra = Racine } \\
\text { TF = Tige Feuillée } \\
\text { Rh = Rhizome }
\end{gathered}
$$

Figure 4. Histogramme des différentes parties utilisées pour la préparation des recettes

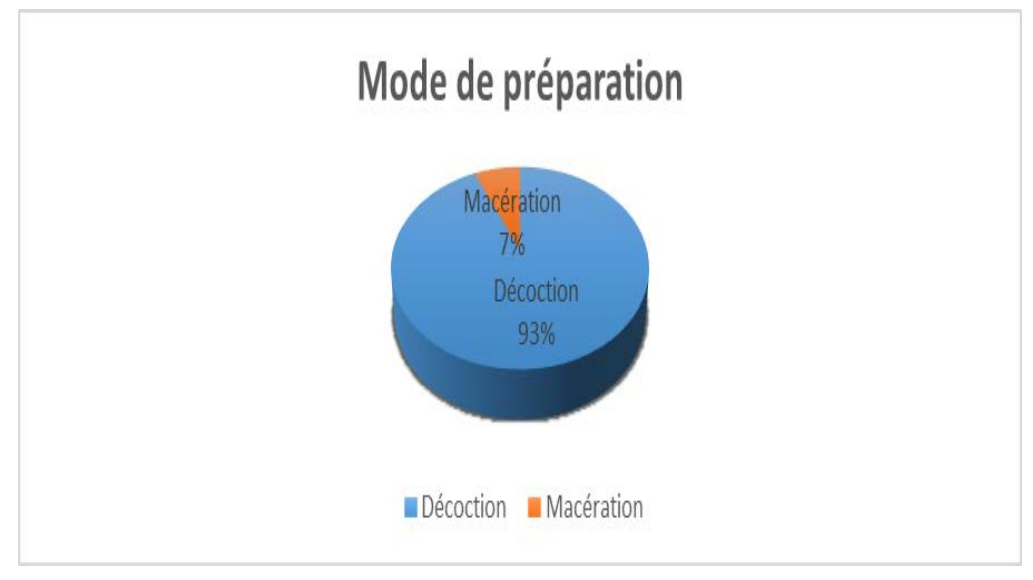

Figure 5. Spectre des différents modes de préparation des remèdes

\section{Espèces sélectionnées pour de futurs tests de laboratoire.}

Par application de la SEPA, Bridelia ferruginea Benth (Euphorbiaceae), Chamaecrista rotundifolia Linn (Leguminosae-Caesalpinioideae) ont été sélectionnées pour les tests de laboratoire sur leurs activités anti-infectieuses (antimicrobiennes).

\section{Discussion}

Ce travail qui est une étude préliminaire réalisée auprès des herboristes de marché (HM) sur les espèces végétales anti-infectieuses, a contribué par enquêtes ethnobotaniques à l'obtention d'une base de données de la Région 
Sanitaire Lomé-Commune du Togo sur cette catégorie de plantes par la méthode togolaise d'enquête ethnobotanique 'ATRM'. Ainsi, cent deux (102) recettes de plantes médicinales anti-infectieuses ont été recensées chez trente-quatre (34) HM à raison de deux (02) par marché soient dix-sept (17) marchés de la zone d'étude. Koudouvo et al., (2011, 2016); Klotoé et al., (2013) ; Dénou et al., (2016) avaient déjà utilisé cette méthode pour recenser des plantes médicinales respectivement au Togo, au Bénin et au Mali pour soigner une diversité de maladies.

L'exclusivité féminine des 34 HB enquêtées qui est une particularité de cette catégorie de praticiens de la Médecine traditionnelle du Togo, a été relatée par des études antérieures (Dougnon, et al., 2018; Koudouvo, 2009; Mehdioui et Kahouadji, 2007). Ceci s'explique par la réalité qu'au Togo le commerce des plantes médicinales est l'apanage des femmes, plus majoritaires que les hommes. Les connaissances endogènes médicinales en Afrique étant transmise de génération en génération, au Togo c'est aux descendants féminins que l'exercice d'herboriste est transmis (Koudouvo et al., 2011 ; Klotoé et al.,2013; Dassou et al.,2014).

Les 68 espèces recensées appartenant à 38 familles botaniques confirment la richesse de la biodiversité médicinale de la "RSLC», les travaux de Koudouvo et al., (2006) ont répertorié en utilisant la même méthode ATRM, 61 espèces antipaludiques dans cette zone. Le nombre d'espèces associé dans chacune des 102 recettes collectées variant de 2 pour les TR à 14 pour les GN est la preuve que les herboristes de marché (HM) disposent réellement des connaissances endogènes pouvant contribuer à la lutte contre les intoxications organiques causées par un nombre très élevé de plantes dans les recettes médicinales du Togo surtout les recettes antiinfectieuses. Ici encore l'un des objectifs fondamentaux de l'ATRM est atteint (Koudouvo et al.,2016 ; Koudouvo 2009). Cependant l'association de 5 espèces et plus dans les recettes relate le manque de diagnostic et les motifs financiers. Les raisons pécuniaires qui sont à la base de l'association en grand nombre d'espèces dans les recettes par les herboristes de marché avaient été relatées par les travaux au Togo sur les recettes antipaludiques (Koudouvo, 2009).

La fréquence la plus élevée de Lannea kerstingii A. Rich s'explique par le fait que la plupart des herboristes l'ont ajouté systématiquement à d'autres plantes pour ses propriétés antianémiques ou pour renfoncer l'organisme et lui permettre de supporter le traitement. Une situation similaire a été signalée par Dougnon et al. (2018) à propos des espèces comme Hibiscus surattensis L, Jatropha gossypifolia Linn. et Sorghum bicolor (Linn.) Moench., alors que l'espèce antiinfectieuse la plus citée dans leur étude a été Bridelia ferruginea Benth. 
Les familles botaniques les plus représentées sont les Annonaceae et Leguminosae -Caesalpinioideae (5 espèces chacunes). Les travaux d'Agody et al.,(2019) infirme ces résultats avec les Caesalpiniaceae et les Euphorbiaceae comme familles prédominantes. Alfa et al., (2018) ont montré quant à eux, une prédominance des Fabaceae. Certaines des plantes de la présente étude se retrouvent dans les travaux de ces deux auteurs. C'est la preuve de la richesse du Togo en matière de plantes médicinales et de connaissances endogène sur ces plantes. Certaines des espèces appartenant à ces familles les plus représentées (Paullinia pinnata, Piliostigma thonningii, et Parkia biglobosa), ont été également recensées au cours des travaux antérieurs (Ladoh-Yemeda et al., 2016 ; Camara et al., 2016) comme étant des plantes anti-infectieuses. Quelques espèces antimicrobiennes au Togo avaient été sélectionnées et testées au laboratoire (Pissang et al, 2016) mais pas en utilisant ATRM pour le choix de ces espèces.

Ecorce de tronc (53,57\%), racine $(16,04 \%)$ et plante entière $(6,65 \%)$ sont entre autres les parties les plus utilisées des espèces recensées. A propos de ces parties, les travaux de Kokou et al., (2000), et de Koudouvo (2009) ont attiré l'attention sur leur prélèvement qui sont source de la menace de la biodiversité. Ambe et al., (2015); Ladoh-Yemeda et al., (2016), Sylla et al., (2018), Alfa et al., (2018), Agody et al., (2019) ont relevé dans leurs travaux, une prédominance des feuilles. Les résultats de cette étude sont proches de ceux de Mpondo et al., (2017) au Cameroun qui ont signalé les écorces (72\%) comme les organes les plus utilisés. Ces mêmes auteurs et Salhi et al., (2010) ont révélé que les arbres ont été le type morphologique majoritaire confirmant les 39,71\% d'arbres comme le type morphologique le plus prépondérant mentionné par les présents travaux. La particularité ethnopharmacologique des résultats de cette étude est d'une part l'administration des recettes à 100\% par voie orale, et d'autre part, la décoction $(93,14 \%)$ et la macération (6,86\%) comme seuls modes de préparation de ces recettes. Les recettes recensées dans des études antérieures (Dénou et al., 2016 ; Koudouvo et al., 2011 et 2016 ; Klotoé et al., 2013 ; Salhi et al., 2010 ; Koudouvo, 2009) ont été préparées et administrées qualitativement par les mêmes modes de préparation et voies d'administration mais à des proportions variables.

Bridelia ferruginea Benth et Chamaecrista rotundifolia (Pers.) ont été sélectionnées par la présente étude afin de confirmer leur propriété antiinfectieuse par de futurs tests de laboratoire.

\section{Conclusion}

La présente étude a permis de mettre en exergue la diversité biologique en plantes médicinales antiinfectieuses au Togo ainsi que la richesse en connaissance endogènes de sa Région Sanitaire de Lomé-Commune. Elle a permis par ailleurs de constituer une base de données de cette catégorie de 
plantes médicinales. Des études complémentaires sont nécessaires dans le futur pour confirmer les activités pharmacologiques avérées de ces plantes en vue de la recherche de molécules pour la mise au point des phytomédicaments améliorés pour la prise en charge des maladies infectieuses au Togo et en Afrique de l'Ouest. A cet effet deux espèces (Bridelia ferruginea et Chamaechrista rotundifolia) ont été sélectionnées.

\section{References:}

1. Aburjai, T., Hudaib, M., Tayyem, R., Yousef, M., Qishawi, M. (2006). Ethnopharmacological survey of medicinal herbs in Jordan, the Ajloun Heights region. Journal of Ethnopharmacology 110 (2007) 294-304

2. Adjanohoun, E.J., Adjakidjè, V., Ahyi, M.R.A., Ake-Assi, L., Akoègninou, A., d'Almeida, J., Apovo, F., Boukef, K., Chadare, M., Cusset, G., Dramane, K., Eymé, J., Gassita, J.N., Gbagidi, N., Goudoté, E., Guinko, S., Houngnon, P., Lo, I., Saadou, M., Sodogandji, Th., De Souza, S., Tchabi, A., Zinsou, D.C., Zohoun, Th. (1989). Contribution aux études ethnobotaniques et floristiques au Bénin. Agence de Coopération Culturelle et Technique, Paris, 895p.

3. Adjanohoun, E.J., Adjakidjè, V., Ahyi, M.R.A., Ake-Assi, L., Akpagana, K., Chibon, P., Al-Hadji, A., Eymé, J., Garba, M., Gassita, J.N., Gbeassor, M., Goudote, E., Guinko, S., Hodouto, K.K., Houngnon, P., Kéita, A., Keoula, Y., Klugu-Ocloo, W.P., Siamevi, K.M., Taffame, K.K. (1986). Contribution aux études ethnobotaniques et floristiques au Togo. Agence de Coopération Culturelle et Technique, Paris, 671p.

4. Agody, M., Bakoma, B., Batawila, K., Wala, K., Dourma, M., Pereki, H., Dimobe, K., Bassene, H., Akpagana, K., (2019). Contribution au recensement des plantes médicinales du Togo: Cas de la Région Maritime. European Scientific Journal 15(24) 329-345.

5. Akouègninou, A., Van der Burg, W.J., Van der Maesen, L.J.G. (2006). Flore analytique du Bénin. Backhuys Publishers, 1043p.

6. Alfa, T., Anani, K., Adjrah, Y., Batawila, K., Ameyapoh, Y. (2018). Ethnobotanical Survey of Medicinal Plants Used Against Fungal Infections in Prefecture of Sotouboua Central Region, Togo. European Scientific Journal, 14, 342-356.

7. Ambe, A. S., Ouattara, D., Tiebre, M. S., Vroh, B. T. A., Zirihi, G. N., N'guessan, K. E. (2015). Diversité des plantes médicinales utilisées dans le traitement traditionnel de la diarrhée sur les marchés d'Abidjan (Côte d'Ivoire). Journal of Animal \& Plant Sciences, 26(2), 4081-4096. 
8. Assouma, A. F., Koudouvo, K., Diatta, W., Vidzro, M.K., Guelly, A.K., Dougnon, J., Agbonon, A., Tozo, K., Gbeassor, M. (2018). Enquête Ethnobotanique Sur La Prise En Charge Traditionnelle De l’Infertilité Féminine Dans La Région Sanitaire

9. Des Savanes Au Togo. European Scientific Journal, Edition. 14 (3) 357-383.

10. Bruneton, J. (1999). Pharmacognosie, Phytochimie, Plantes médicinales. Ed. medicales internationales Editions Technique \& Documentation, Cachan, [S.l.], , p. 647-673

11. Camara, D., Bene, K., Gnahoue, G., Zirihi, G. N. (2016). Etude Ethnobotanique, Evaluation De L'activite Antifongique Sur Candida Albicans Et De La Toxicite Sur Des Cellules Hff De Bersama Abyssinica (Fresen.), Une Plante De La Pharmacopee Ivoirienne. European Scientific Journal, 12(3) :171-185

12. Courvalin, P. (1997). Evolution de la résistance aux antibiotiques. Éditorial, Médecine/Sciences 1997; 13: 925-6.

13. Cragg, M. G., Newman, J.D. (2002). Drugs from nature: past achievements, future prospects. Advance in Phytomedecine. Ethnomedicine and drug discovery 01, 23-37.

14. Dassou, H. G., Ogni, C. A., Yédomonhan, H., Adomou, A. C., Tossou, M., Dougnon, J. T., Akoègninou, A. (2014). Diversité, usages vétérinaires et vulnérabilité des plantes médicinales au NordBénin. International Journal of Biological and Chemical Sciences, 8(1), 189-210.

15. Dénou, A., Koudouvo, K., Togola, A., Aziati, K.Y., Esseh, J., Ajavon, C.A., Essien, K., Aklikokou, K., Sanogo, R., Diallo, D., Gbeassor, M. (2016). Traditional knowledge on antimalarial plants having analgesic properties, used in Togo Maritime Region. The Journal of Ethnobiology and Traditional Medicine. Photon 126(2016) 11601170.

16. DGSCNT, 2011. Recensement de la population et de l'habitat du Togo, 2010, 162p.

17. Diallo, A., Gbeassor, M., Vovor, A., Eklu G, K., Aklikokou, K. Agbonon, A. (2008). Effect of Tectonagrandis on phenylhydrazine induced aneamia in rats: Fitoterapia, 79: 332-336.

18. Dieye, P. I., Sarr, S. O. (2020). État de la recherche de molécules cibles antimicrobiennes issues de plantes en Afrique. Afrique SCIENCE, 16(1), 348-374.

19. Dougnon, V., Legba, B., Yadouléton, A., Agbankpe, J., Koudokpon, H., Hounmanou, G., Amadou, A., Fabiyi, K., Assogba, P., Hounsa, E., Aniambossou, A., Déguenon, E., de Souza, M., Bankolé H.S., Dougnon, J., Baba-Moussa, L. (2018). Utilisation des plantes du Sud- 
Bénin dans le traitement de la fièvre typhoïde: rôle des herboristes. Ethnopharmacologia, 60 (11) :60-74

20. Eklu-Natey, R.D., et Balet A., (2012). Pharmacopée africaine. Dictionnaire et monographies multilingues du potentiel médicinal des plantes africaines : Afrique de l'ouest. Volume 1, Lausanne: Éditions d'en-bas; Genève: Traditions et Médecine, 912p.

21. Esseh, K., Afanyibo, Y.G., Ahama-Esseh, K.Y.S., Idoh, K., Koudouvo, K., Agbonon, A., Gbeassor, M. (2019). Screening Phytochimique, Étude Toxicologique, Évaluation des Activités Antiplasmodiale et Antiradicalaire de la Tige Feuillée de Senna occidentalis Linn (Fabaceae). European Scientific Journal February 2019 edition 411(15) : 411-433

22. Gazzaneo, L., de Lucena, R., Albuquerque, U. 2005. Knowledge and use of medicinal plants by Local specialists in a region of Atlantic Forest in the state of Permanbuco (Northeastern Brazil). Journal of Ethnopharmacology and Ethnomedicine 1, 1-8.

23. Klotoé, J. R., Dougnon, T. V., Koudouvo, K., Atègbo, J. M., Loko, F., Akoègninou, A., Gbeassor, M. (2013). Ethnopharmacological survey on antihemorrhagic medicinal plants in South of Benin. European Journal of Medicinal Plants, 3(1), 40-51.

24. Kokou, K., Caballé, G., 2000. Les Îlots forestiers de la plaine côtière togolaise. Bois et Forêts des Tropiques, № 263 (1). 3951.

25. Koudouvo, K., A. Denou, K. Asseh, R., Sanogo, K., Essien, D., Diallo, K., Kokou, S. K., Tozo, K., Aklikokou, J. C., Aguiyi, C., Gbéassor, M. (2017). Ethnobotanical Survey of Endangered Antimalarial and Analgesic Plants of Togo for the Safeguard of the Medicinal Biodiversity. Journal of Agriculture and Ecology Research International 12(2):1-9.

26. Koudouvo, K., Esseh K., Denou A., Aziati T., Ajavon C., Afanyibo, Y.G., Agbonon A., Sanogo R., Dougnon J., Aklikokou, K., Aguiyi, J.C., Diallo, D., Mensah, G.A., Gbeassor, M. (2016). Ethnopharmacological study of antimalarial recipes in Togo for a formulation of phytomédecine for malarial taking care. Bulletin de la Recherche Agronomique du Bénin (BRAB) Numéro 79: 1025-2355.

27. Koudouvo, K., Karou, D.S., Kokou, K., Essien, K., Aklikokou, K., Glitho, I.A., Simpore, J., Sanogo, R., De Souza, C., Gbeassor, M. (2011). An ethnobotanical study of antimalarial plants in Togo Maritime Region. Journal of Ethnopharmacology 
134 (2011) 183-190.

28. Koudouvo, K. (2009). Contribution à la recherche sur les plantes médicinales à propriété antipaludique du Togo. Thèse de Doctorat Unique de l'Université de Lomé en Biologie de Développement. Option: Ethnobotanique et Pharmacologie des Substances Naturelles, 182p.

29. Koudouvo, K., Kavegue, A., Agbonon, A., Kodjo, A., Aklikokou, K., Kokou, K., Essien, K., Gbeassor, M. (2006). Enquête ethnopharmacologique sur les plantes à activité antiplasmodiale, antioxydante et immunostimulante dans la Région Maritime du Togo. Revue Togolaise des Sciences Vol. 1, pp 144-155.

30. Ladoh-Yemeda, C. F., Vandi, T., Dibong, S. D., Mpondo, E. M., Wansi, J. D., Betti, J. L., Choula, F., Ndongo, D., Eyango, M. T. (2016). Étude ethnobotanique des plantes médicinales commercialisées dans les marchés de la ville de Douala, Cameroun. Journal of Applied Biosciences, 99(1), 9450-9466.

31. Leonti, M., Stafford, G.I., Dal, C.,M., Cabras, S., Castellanos M.E.,Casu L., Weckerle, C.S. (2017). Reverse ethnopharmacology and drug discovery. Journal of Ethnopharmacology, 198, 417-431.

32. Mehdioui, R., Kahouadji, A. (2007). Etude ethnobotanique auprès de la population riveraine de la forêt d'Amsittène: cas de la Commune d'Imi n'Tlit (Province d'Essaouira). Bulletin de l'Institut Scientifique Rabat, section Sciences de la Vie, 29, 11-20.

33. Mpondo, M. E., Vandi, D., Ngouondjou, F. T., Mvogo, O. P. B., Embolo, E. E., et Dibond, S. D. (2017). Contribution des populations des villages du centre Cameroun aux traitements traditionnels des affections des voies respiratoires. Journal of Animal \& Plant Sciences, 2017. 32(3): 5223-5242.

34. PAO : Pharmacopée d'Afrique de l'Ouest, 2013. Organisation Ouest Africaine de la Santé (OOAS), Edition $\mathrm{N}^{\circ} 1,257 \mathrm{p}$.

35. Pissang, P., Agban, A., Yao, P. H., Tchacondo, T., Sadji, A. Y., Effoe, S., Batawila, K. (2016). Evaluation In Vitro De L'activité Antimicrobienne Des Extraits De Cassia alata Linn. (Fabaceae). European Scientific Journal, 12(21).

36. Salhi, S., Fadli M., Zidane L., Douira A. (2010). Etudes floristique et ethnobotanique des plantes médicinales de la ville de Kénitra (Maroc). Lazaroa, 31, 133-146.

37. Soh, N.P., Benoit-Vical, F. (2007). West African plants, a source of future antimalarial drugs. Journal of ethnopharmacology 114, 130140.

38. Sylla, Y., Silue, D. K., Ouattara, K., Kone, M. W. (2018). Etude ethnobotanique des plantes utilisées contre le paludisme par les 
tradithérapeutes et herboristes dans le district d'Abidjan (Côte d'Ivoire). International Journal of Biological and Chemical Sciences, 12(3), 1380-1400.

39. PROTA4U: www.prota4u.org /07/2019 